\title{
Performances of Vertical Handovers for Multimedia Traffic between WLAN, WiMAX and 3G Mobile Networks
}

\author{
Kire Jakimoski \\ Ss. Cyril and Methodius University, Faculty of \\ Electrical Engineering and Information Technologies, \\ Karpos 2 bb, 1000 Skopje, Macedonia \\ kire_jakimoski@yahoo.com
}

\author{
Toni Janevski \\ Ss. Cyril and Methodius University, Faculty of \\ Electrical Engineering and Information Technologies, \\ Karpos 2 bb, 1000 Skopje, Macedonia \\ tonij@feit.ukim.edu.mk
}

\begin{abstract}
The effect of the terminal speed and traffic type on the detection and selection process of the preferred access network in the IEEE 802.21 is not yet well understood. Efficiency of vertical handovers depends on the appropriate QoS of the users using mobile devices. One of the most important QoS parameters is the throughput between the base stations and the mobiles.

The focus of this paper is to show how IEEE 802.21 standard for media - independent handover services supports mobility and traffic type (voice, video) between UMTS, WiMAX and WLAN networks. In particular, duration of the vertical handover process between UMTS, WiMAX and WLAN is examined (using different mobile terminal speed and traffic type). In order to test the efficiency of vertical handovers, throughput performance during the vertical handovers between heterogeneous networks is also researched in this paper. A novel method for analyzing the vertical handover performance is invented and new conclusions are presented from the simulation results.
\end{abstract}

\section{Keywords}

IEEE 802.21, Quality of Service, UMTS, Vertical Handover, WiMAX (IEEE 802.16), WLAN (IEEE 802.11).

\section{INTRODUCTION}

One of the challenging problems for coordination within a diverse-network environment is vertical handover, which is the decision for a mobile node to handoff between different types of networks [1]. The purpose of IEEE 802.21 standard is to improve the user experience of mobile devices by facilitating handover between 802 networks whether or not they are of different media types and to make it possible for mobile devices to perform seamless handover [2].

Various vertical handoff decision algorithms have been proposed recently [3]. A predictive link triggering mechanisms for seamless handover in heterogeneous networks are also proposed $[4,5]$ to improve handover performance in IEEE 802.21 standard. The effect of mobile terminal speed on WLAN/3G vertical handovers

Permission to make digital or hard copies of all or part of this work for personal or classroom use is granted without fee provided that copies are not made or distributed for profit or commercial advantage and that copies bear this notice and the full citation on the first page. To copy otherwise, or republish, to post on servers or to redistribute to lists, requires prior specific permission and/or a fee.

Mobimedia'09, September 7-9, 2009, London, UK. Copyright 2009 ICST 978-963-9799-62-2/00/0004 ... \$5.00". is analyzed in [6]. Handover latency for the cases where UDP and TCP carry MIH signalling messages is modelled and some of the design tradeoffs are discussed in [7].

In order to continue with this progress, the authors of this paper are focused on the duration of vertical handovers between UMTS/WiMAX/WlAN networks and throughput performance during these vertical handovers under different mobile terminal speed and traffic type. Motivation for this paper is exactly this field of researching. Thorough analysis of the vertical handover duration and throughput performance during the vertical handover process has been done using ns-2 simulator in linux environment [8]. Furthermore, the effect of mobile terminal speed and traffic type (voice, video) is analyzed for vertical handovers between UMTS, WiMAX and WLAN networks.

During the analysis of the simulation results, a novel method for evaluating vertical handover performance has been used. In brief, the method consists of presenting the instant throughput results every $100 \mathrm{~ms}$ during the vertical handover process and comparing them with the vertical handover duration results. The time of initiation and ending of the throughput degradation/disruption during the vertical handover process is compared with the time of initiation and ending of the vertical handover process.

Section II presents the simulation environment and parameters that have been used for the simulations. Section III shows the results from the simulations in order to analyze the performance of the different networks in the vertical handover process. We summarize our results in Section IV.

\section{SIMULATION ENVIRONMENT}

Simulation study in this paper was performed using ns-2 simulator with the mobility package tool from NIST. Scenario consists three access points (base stations) for UMTS, WiMAX and WLAN network configured on a $2000 \times 2000$ meters topography. IEEE 802.11 (WLAN) access point has $\mathrm{x}=800$ meters, $\mathrm{y}=1000$ meters coordinates in the network, IEEE 802.16 (WiMAX) base station has $\mathrm{x}=1100$ meters, $\mathrm{y}=1000$ meters coordinates and UMTS base station covers the whole simulated area. Mobile terminal node starts to move from $x=400, y=1000$ meters coordinates to $x=1900$, $y=1000$ meters coordinates. The speed of the mobile terminal node in the simulations changes from $10 \mathrm{~km} / \mathrm{h}$ to $120 \mathrm{~km} / \mathrm{h}$ with intervals of $10 \mathrm{~km} / \mathrm{h}$.

Simulations have been done using modified version of ns-2.29 which contains an implementation of MIHF (Media Independent Handover Function) based on the draft of IEEE 802.21 
specifications [9]. Simulation parameters that are used for neighbor discovery and application traffic are:

- Neighbor Discovery: - RA interval: U[200s,600s], max delay between RA: $0.5 \mathrm{~s}$, router lifetime: $1800 \mathrm{~s}$;

- Application traffic (UDP): - video streaming (320kbps, $4 \mathrm{pkt} / 100 \mathrm{~ms}, 1240$ bytes), voice traffic (64kbps, packet size 1500 bytes).

Parameters that have been used for IEEE 802.16 and IEEE 802.11 networks during the simulations are:

- IEEE 802.16 parameters: - coverage: $500 \mathrm{~m}$ radius, DCD/UCD interval: $5 \mathrm{~s}$, frame duration: $4 \mathrm{~ms}$, contention opportunity per frame: 5 , backoff window size start for contention: 2 , scanning: duration $=50$ frames, interleaving $=50$ frames, iteration $=2$;

- IEEE 802.11 parameters: coverage: $50 \mathrm{~m}$ radius, beacon interval: $100 \mathrm{~ms}$.

\section{SIMULATION RESULTS}

\subsection{Vertical Handover Process and}

\section{Throughput Performance}

Fig. 1 shows completely the process of handover between the UMTS and WiMAX network when simulations were executed. Every step of the handover process has some duration, so equation (1) calculates the duration of handover like a sum of the duration of every step of the handover process.

$$
\begin{aligned}
& H=\tau_{L U}+ \tau_{R S}+\tau_{R A}+\tau_{R M}+\ldots \\
& \ldots+\tau_{A C K}+\tau_{R E Q}+\tau_{R E S}
\end{aligned}
$$

where $\tau_{\mathrm{LU}}$ is part of time from the vertical handover process from initiation of handover process when link is detected in multiface node 5.0.0. to the moment when handover received link up in the multiface node; $\tau_{\mathrm{RS}}$ is the time spent for sending router solicitation (RS) from the multiface node to the base station $4.0 .0 ; \tau_{\mathrm{RA}}$ is the time needed for router advertisement (RA) received from ND module in the interface $4.0 .1 ; \tau_{\mathrm{RM}}$ is the time for the redirect message to traverse from the multiface node to the router 1.0.0; $\tau_{\mathrm{ACK}}$ is the time for the acknowledge for redirect packet to traverse from the router to the multiface node; $\tau_{\mathrm{REQ}}$ is the time for MIH Agent in the multiface node to send capability discovery request to the base station 4.0.0 and $\tau_{\mathrm{RES}}$ is the time for receiving capability discovery request in the multiface node from the base station. The vertical handover process between WiMAX and WLAN network has completely the same steps as in Fig. 1 except that the interface is 3.0.1 and the base station is 3.0.0 in the simulations.

Fig. 2 shows the vertical handover process between WLAN and WiMAX presenting every step of the handover and its duration. Equation 2 gives the duration of this handover process as a sum of the steps of the handover:

$$
H=\tau_{R M}+\tau_{A C K}
$$

where $\tau_{\mathrm{RM}}$ is the time needed for sending redirect message from the multiface node 5.0.0 in Fig. 5 to the router 1.0.0 and $\tau_{\mathrm{ACK}}$ is the time needed for acknowledgment for redirecting packet traversed from the router to the multiface node. WiMAX/UMTS vertical handover has the same handover process steps as shown in Fig.2, except that the interface is 0.0 .2 and the base station is 0.0 .1 .

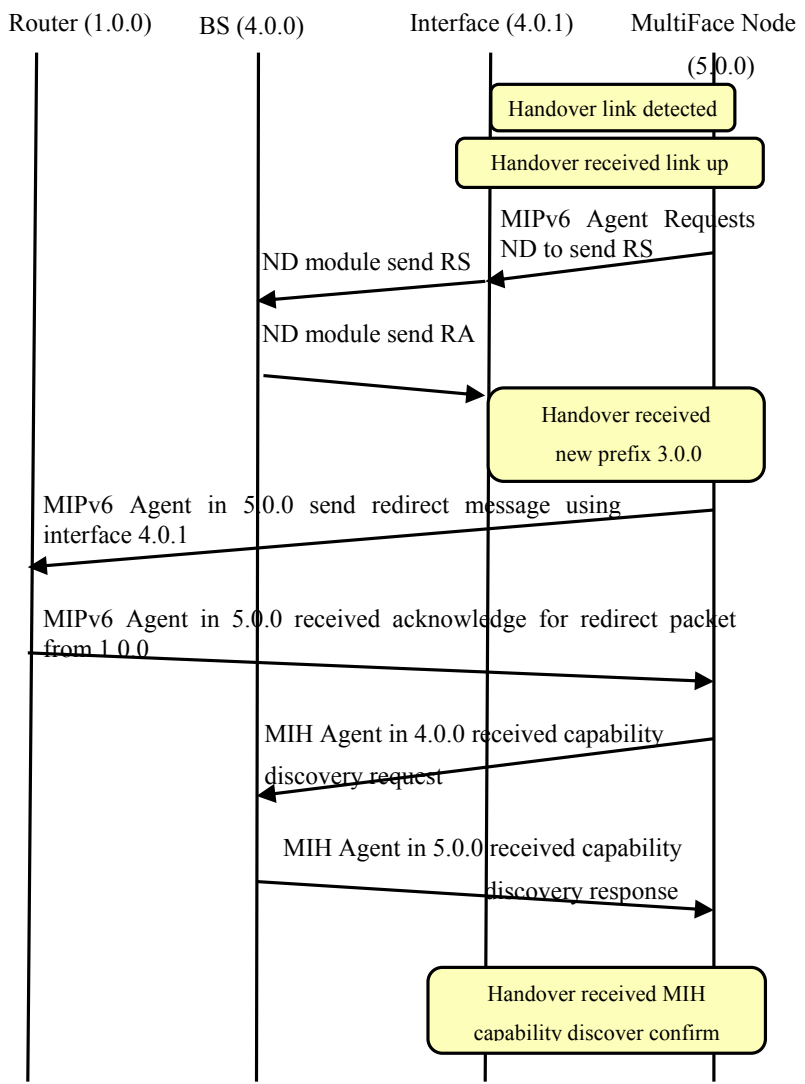

Figure 1. Vertical Handover flow between UMTS and WiMAX

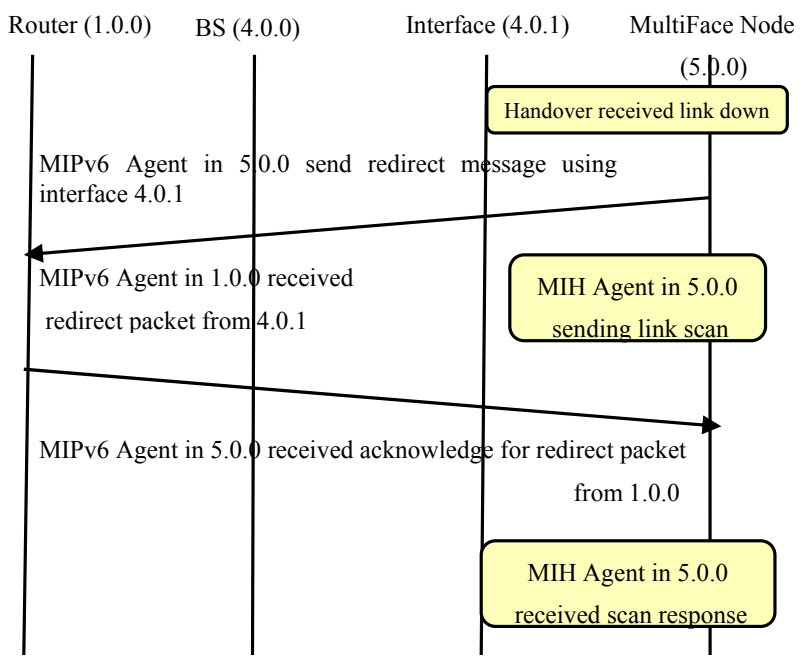

Figure 2. Vertical Handover flow between WLAN and WiMAX 


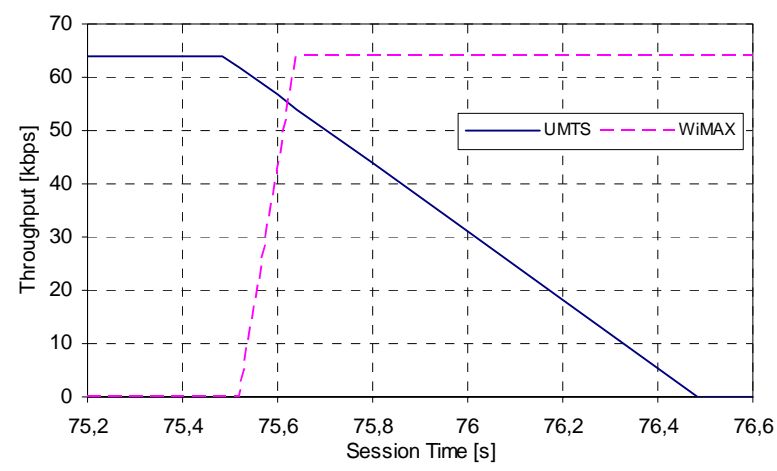

a. Throughput of the UMTS-WiMAX vertical handover

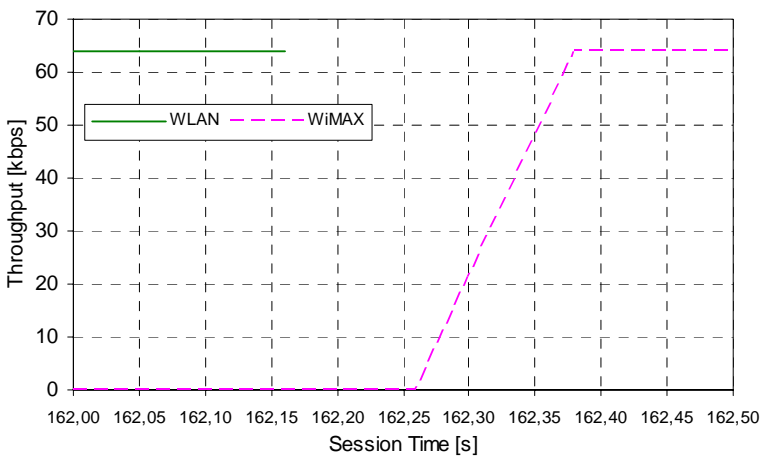

b. Throughput of the WLAN-WiMAX vertical handover

Figure 3. Throughput for voice traffic and $10 \mathrm{~km} / \mathrm{h}$ speed of the mobile terminal

Fig. 3.a shows the throughput performance during the vertical handover process when the both networks are in range during the handover process (make before break). This occurs with UMTS/WiMAX and WiMAX/WLAN vertical handovers in our simulations. Fig. 3.b presents the throughput performance during the vertical handover process when the first network is out of range during the vertical handover process and only the second network is available (break before make). This happens with WLAN/WiMAX and WiMAX/UMTS vertical handovers here.

This part of the simulation results researches the throughput performance and vertical handover process steps. Results show that vertical handover process steps and throughput performance differs for the two types of vertical handovers (when two networks are available and only one network is available during the vertical handover process). Throughput gap during the handover process lasts approximately same, but duration of vertical handover process differs a lot between the two types of vertical handovers. Duration is much lower for the second type of vertical handover process. This can be seen from the steps in Fig. 1 and Fig. 2 of the vertical handover process.

\subsection{Vertical Handover Duration}

Fig. 4 presents the results from the simulations about the duration of the vertical handover process explained in Fig. 1 and Fig. 2.

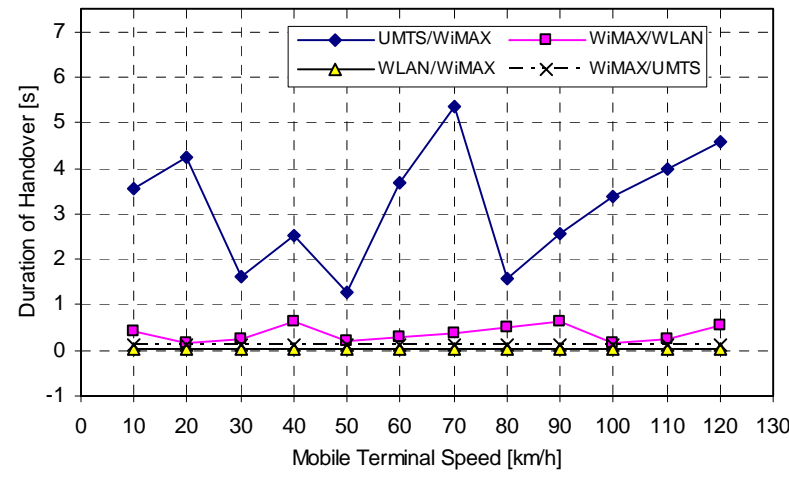

Figure 4. Duration of vertical handover between UMTS, WiMAX and WLAN for voice traffic

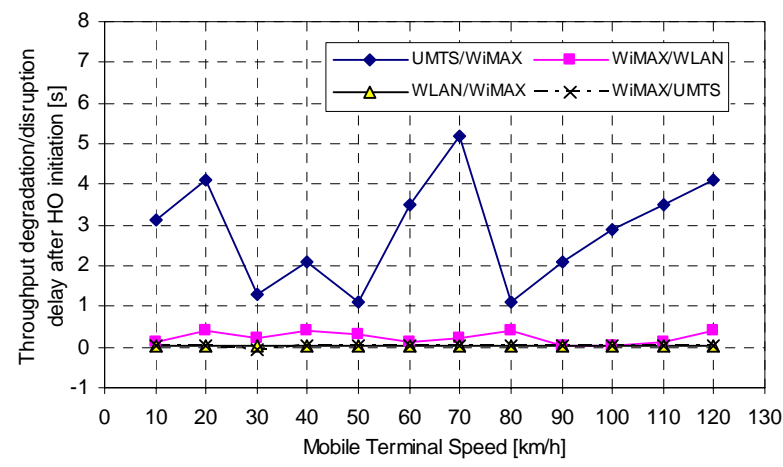

Figure 5. Throughput degradation/disruption delay after vertical HO initiation for video traffic

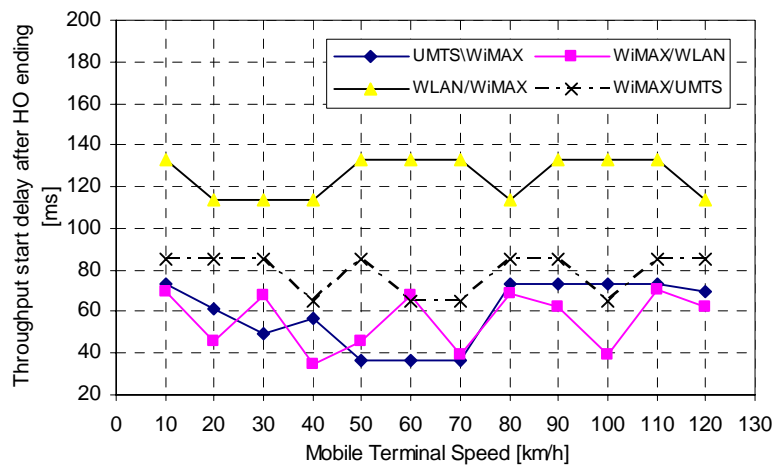

Figure 6. Throughput establishment delay after vertical HO ending for voice traffic

This duration of the vertical handover is the sum $\mathrm{H}$ in the equations 1 and 2 for the two types of vertical handovers.

Duration of vertical handover process is analyzed for the two types of vertical handovers, changing the type of traffic (voice, video) and the mobile terminal speed. Comparing the durations of vertical handover, the results show that mobile terminal speed has impact on the first type of vertical handover, but has no impact or that impact is negligible for the second type of vertical handover. 
This impact of the mobile terminal speed doesn't increase the values for duration of the vertical handover as speed increases. The curve of duration of vertical handover has ups and downs in some limits. The impact is the same when comparing voice and video traffic, with one note that video traffic has lower values for vertical handover duration than voice traffic when comparing mobile terminal speeds from 10 to $120 \mathrm{~km} / \mathrm{h}$.

\subsection{Throughput during Vertical Handovers}

Now, we'll analyze performance of the throughput between the networks during the vertical handover process. Different types of vertical HO between different types of networks are included in the investigations changing the mobile terminal speed and traffic types.

Fig. 5 shows delays of starting the throughput degradation or disruption after the initiation of the vertical handover process for video traffic and for different types of vertical handovers, changing the mobile terminal speed.

Fig. 6 presents that the highest throughput establishment delay has WLAN/WiMAX vertical HO process, then WiMAX/UMTS vertical HO. UMTS/WiMAX and WiMAX/WLAN has lower variable values for different mobile terminal speeds in approximately same range of delay in ms. Because of saving space reasons, graphic results for throughput degradation/disruption delay after vertical HO initiation for voice traffic and for throughput establishment delay after vertical $\mathrm{HO}$ ending for video traffic are not presented here.

This part of the researches in this paper analyses delay of throughput degradation or disruption after vertical HO initiation and delay of throughput establishment after vertical HO ending for different type of vertical handover, changing the mobile terminal speed and the traffic type (voice, video). Results show that delay of throughput degradation after vertical $\mathrm{HO}$ initiation is much higher for the first type of vertical handover and has variable results as mobile terminal speed increases. And the delay of throughput disruption after vertical HO initiation is much lower for the second type of vertical handover and the variations as mobile terminal speed increases are negligible compared with the first type of vertical handover. Delay of throughput degradation or disruption is a bit lower for video traffic compared with voice traffic.

The results presented in Fig. 6 are opposite about the delay of throughput establishment after vertical HO ending. Here delay of throughput establishment after vertical $\mathrm{HO}$ ending is lower for the first type of vertical handover compared with the second type of vertical handover. This happens because the vertical handover duration of the second type of vertical handover is much smaller than the first type of vertical handover. The results of the delay of throughput establishment after vertical HO ending are variable as mobile terminal speed increases in some min and max limits for voice traffic. Mobile terminal speed has no impact on the delay of throughput establishment after vertical HO ending between WLAN/WiMAX networks for video traffic. Comparing voice and video traffic about the delay of throughput establishment after vertical HO ending, we can conclude that this delay is a little higher for video traffic and more dependable from the mobile terminal speed for voice traffic.

\section{CONCLUSION}

Having comparative analysis of the results for vertical handover duration and throughput delay after vertical HO initiation and ending for different types of vertical handovers under different mobile terminal speed, this paper gives a thorough analysis of the vertical handover processes in IEEE 802.21 standard. Given conclusions will help the researchers better to understand the correlations between the vertical handover duration, throughput performance and delay during the handover process and dependence of these parameters from the traffic type (voice, video) and mobile terminal speed.

Given results will also help researchers to improve the algorithms for vertical handover process in order to reduce the duration of vertical handover process and throughput gap between the networks in order customers to have better QoS using IEEE 802.21 standard in the future. A method that is used in this paper is novel and can help researches to test their improvements of the duration of handover process and throughput performance during and after handover process. This paper also may give new ideas for future simulations with different scenarios which may include different traffic types and topology scenarios.

\section{REFERENCES}

[1] F. Zhu, J McNair, "Multiservice Vertical Handoff Decision Algorithms", Wireless \& Mobile Systems Laboratory, Department of Electrical and Computer Engineering, University of Florida, May, 2006.

[2] S.J. Yoo, D. Cypher, N. Golmie, "Timely Effective Handover Mechanism in Heterogeneous Wireless Networks", Gaithersburg, MD 20899-8920, USA.

[3] Enrique Stevens-Navarro, "A Vertical Handoff Decision Algorithm in Heterogeneous Wireless Networks", Second UBC-IEEE Workshop on Future Wireless Systems, March $9^{\text {th }}, 2007$.

[4] S.J. Yoo, D. Cypher, and N. Golmie, "Predictive Link Trigger Mechanism for Seamless Handovers in Heterogeneous Handovers,"accepted to appear in Wiley Wireless Communications and Mobile Computing 2008.

[5] S.J. Yoo, D. Cypher, N. Golmie, "LMS Predictive Link Triggering for seamless handovers in heterogeneous wireless networks,"in the Proceedings of MILCOM 2007, Orlando Florida, October 28-30, 2007.

[6] T. Melia, A. de la Oliva, I. Soto, C. J. Bernardos, A. Vidal, "Analysis of the effect of mobile terminal speed on WLAN/3G vertical handovers", Institute of Informatics, University of Goettingen, Germany, 2006.

[7] D. Griffith, R. Rouil, N. Golmie, "Performance Metrics for IEEE 802.21 Media Independent Handover (MIH) Signaling", National Institute of Standards and Technology, Gaithersburg, MD 20899 USA.

[8] Mobility package for NS-2, DOI=http://www.andt.nist.gov.

[9] IEEE P802.21/D11.0, "Draft Standard for Local and Metropolitan Area Networks: Media Independent Handover Services", May, 2008. 